\title{
Hidrocarburos y compensaciones por pérdida de biodiversidad: oportunidad para el desarrollo sostenible
}

\author{
Hydrocarbons and Biodiversity Offsets: An Opportunity for Sustainable Development
}

\author{
Adriana Soto $^{(1)}$, Mariana Sarmiento ${ }^{(2)}$ \\ (1) Economista, Universidad de los Andes. Viceministra de Ambiente y Desarrollo Sostenible 2011-2013. Asesora Senior de Wildlife Con- \\ servation Society para Latinoamérica. Bogotá, Colombia. asoto@wcs.org \\ (2) Magíster en Gerencia Ambiental, Yale University. Coordinadora Negocios y Conservación, Wildlife Conservation Society. Bogotá, Co- \\ lombia.msarmiento@wcs.org
}

Recibido 24 de junio de 2014. Modificado 27 de junio. Aprobado 30 de junio de 2014.

\author{
Palabras clave \\ Compensaciones ambientales, Colombia, \\ licencias ambientales, compensaciones por pérdida \\ de biodiversidad, bancos de hábitat.
}

\section{Resumen}

El Manual para la Asignación de Compensaciones por Pérdida de Biodiversidad adoptado por el Ministerio de Ambiente y Desarrollo Sostenible de Colombia representa un gran avance en la estandarización del cálculo de cuánto, dónde y cómo compensar aquellos impactos sobre la biodiversidad que no pueden ser evitados, mitigados o corregidos por un proyecto licenciado. Además de contribuir a la mitigación de impactos, esta nueva herramienta ofrece una oportunidad para las empresas de hidrocarburos ya que les permite anticipar las medidas de compensación y así planear las estrategias de implementación y financiación. Sin embargo, aún hay retos legales, institucionales, financieros y técnicos para su adecuada implementación. Estos deben superarse para que este esquema innovador de compensaciones pueda asegurar que las inversiones en compensaciones ambientales asociadas a, por ejemplo, la explotación de hidrocarburos, contribuyan de manera efectiva a la metas de conservación de biodiversidad y restauración ecológica del país.

\section{Key words}

Compensatory mitigation, Colombia, environmental

permits, biodiversity offsets, habitat banks.

\begin{abstract}
The Manual for Calculating Biodiversity Offsets adopted by the Ministry of the Environment and Sustainable Development in Colombia is a breakthrough towards the standardization of the assessment of environmental impacts that cannot be avoided, mitigated or corrected during the lifespan of a development project. In addition to contributing to the mitigation of impacts, this new tool offers an opportunity for oil and gas companies to anticipate their compensation requirements and to plan adequate implementation and funding strategies. However, there are still legal, institutional, financial, and technical challenges for its implementation. Overcoming these challenges is key so that the investments in biodiversity offsets associated with this innovative compensation scheme-in this case related to the exploitation of hydrocarbons - contribute effectively to the national goals of biodiversity conservation and ecological restoration.
\end{abstract}

\section{INTRODUCCIÓN}

Colombia ocupa el tercer lugar a nivel mundial en nuevos descubrimientos de petróleo y gas (Asociación Nacional de Hidrocarburos [ANH], 2014) y se espera que la producción de petróleo aumente a un millón doscientos mil barriles de petróleo equivalente al día en el año 2020, en un escenario de proyección moderado (Unidad de Planeación Minero Energética [UPME], 2012). Lo anterior genera una serie de retos para que un sector que jalona la economía, no lo haga en detrimento del capital natural del país, y por lo tanto de su desarrollo sostenible.
Respondiendo a esta necesidad, el Ministerio de Ambiente y Desarrollo Sostenible (MADS) estableció, mediante Resolución 1517 del 31 de agosto de 2012, el Manual para la Asignación de Compensaciones por Pérdida de Biodiversidad (MACPB). Dicho Manual entró en vigencia a partir del 1 de enero de 
2013 y es de obligatorio cumplimiento para una serie de proyectos que requieren y soliciten licencias ambientales, tales como los de explotación de hidrocarburos, ante la Autoridad Nacional de Licencias Ambientales (ANLA).

El MACPB representa un gran avance en la determinación estandarizada y objetiva de cuánto compensar en términos de área, dónde realizar la compensación y las estrategias sobre cómo hacerlo, para aquellos impactos a la biodiversidad que no fueron evitados, mitigados o corregidos en proyectos licenciados ante la ANLA. También abre la posibilidad de que haya un flujo constante y adecuado de recursos para el cumplimento de las metas de conservación y de restauración ecológica que tiene el país y que por ahora dependen en gran medida del presupuesto general de la nación. Sin embargo, dieciocho meses después de su entrada en vigencia, aún hay retos legales, institucionales, financieros y técnicos para que el Manual pueda ser efectivamente implementado.

Teniendo en cuenta lo anterior, este documento presenta los antecedentes y desarrollos normativos de las compensaciones por pérdida de biodiversidad, los desafíos existentes en relación a su implementación, y las lecciones aprendidas de otros países en esta materia. Al final se proponen aproximaciones que podrían contribuir a que las compensaciones por pérdida de biodiversidad se lleven a cabo de manera efectiva. Este artículo retoma algunas de las conclusiones del reciente evento realizado en la Universidad de los Andes: "Diálogos de Biodiversidad. El Futuro de las compensaciones ambientales en Colombia”, así como de la Cumbre Mundial de No Pérdida Neta, realizada en junio de este año en Londres.

\section{AnTECEDENTES Y AVANCES}

El fin último de las compensaciones es lograr que, durante y al final del desarrollo de un proyecto sujeto a licenciamiento ambiental, haya una ganancia neta de biodiversidad o como mínimo una no pérdida neta de la misma (Business and Biodiversity Offsets Programme [BBOP], 2012). Las compensaciones pueden además contribuir al reto financiero que tiene el país para cumplir con las metas de crear y sanear el sistema de áreas protegidas en ecosistemas clave ${ }^{1}$ y a cubrir el déficit de restauración de áreas degradadas o deforestadas ${ }^{2}$. Cumpliendo con las anteriores metas se lograría además revertir la pérdida de servicios ecosistémicos como la regulación hídrica y de suelos, claves para el buen desempeño de otros sectores como el agropecuario, el hidroeléctrico y el sistema de acueductos ${ }^{3}$.

El requerimiento de realizar compensaciones ambientales existe desde hace más de veinte años en Colombia y está enmarcado en cuatro instrumentos: la licencia ambiental, la sustracción de áreas de reserva forestal, el aprovechamiento forestal y la compensación por aprovechamiento de especies amenazadas (Sarmiento, López y Mejia, 2014). Para las actividades del sector de hidrocarburos, dependiendo de las características del proyecto y su ubicación, el esquema de compensaciones puede resultar de una combinación de los diferentes instrumentos antes mencionados.

En el caso de la compensación ambiental asociada al licenciamiento ambiental, ésta se estableció desde la Constitución de 1991, y fue posteriormente reglamentada por la Ley 99 de 1993 y el Decreto 2820 de 2010. Hasta el 2012, previa la expedición del MACPB, faltaba claridad metodológica y de criterios técnicos y científicos para determinar objetivamente la valoración y asignación de las compensaciones. Lo anterior llevaba a que las compensaciones se enfocaran en actividades de reforestación comercial con especies foráneas, en áreas que podían variar entre 1 y 400 hectáreas sin considerar la integralidad de los ecosistemas y la pérdida de biodiversidad derivada de los impactos ambientales de la actividad licenciada. Adicionalmente, en un estudio realizado por la Wildlife Conservation Society (WCS), en convenio con el Ministerio de Ambiente, se observó que, de 167 expedientes revisados en diez Corporaciones Autónomas Regionales (CAR), el 61\% no especificaba la duración de las medidas de compensación y el 30\% tenía una duración de entre uno y tres años. Esto en comparación con la duración del proyecto que en la mayoría de las licencias ambientales, era entre 25 y 30 años (WCS, 2013).

Como consecuencia de la falta de claridad en la forma de calcular y asignar las compensaciones, no se generaba una relación entre la afectación causada al ecosistema y las medidas de compensación impuestas (Sáenz, Walschburger, González, McKenney y Kiesecker, 2013). Producto de lo anterior, en algunas ocasiones, cuantiosas inversiones en compensaciones hechas por el proyecto licenciado terminaban perdiéndose y en muchos casos se generaba un costo reputacional para la empresa al no visibilizarse la compensación efectiva de sus impactos en la biodiversidad o en los ecosistemas del área del proyecto.

Teniendo en cuenta lo anterior, la Resolución 1503 de 2010 y la Ley 1450 de 2011 establecieron la necesidad de

\footnotetext{
1 Actualmente, más de la mitad de los ecosistemas naturales terrestres del país no se encuentran adecuadamente representados en el Sistema Nacional de Áreas Protegidas (documento sin publicar).

2 Aunque la tasa promedio anual de deforestación para el periodo 2011-2012 llegó a aproximadamente 296.000 hectáreas (ha) y disminuyó con respecto al periodo 1990-2010 donde la tasa promedio estaba en 310.000 ha/año, solamente se alcanzan a restaurar 90.000 ha anualmente (MADS, 2013).

3 De acuerdo con el IDEAM, los índices de escasez hídrica más altos del país se encuentran justo en las áreas donde hay menor cobertura natural y donde se ubican la mayor parte de la población y de la actividad económica del país.
} 
mejorar y estandarizar los mecanismos de compensación ambiental a través de metodologías, criterios y procedimientos desarrollados por el Ministerio de Ambiente. Así mismo, la Política para la Gestión Integral de la Biodiversidad y sus Servicios Ecosistémicos (PNGIBSE), aprobada por el Ministerio en 2012, estableció como uno de sus ejes estratégicos fortalecer la evaluación y asignación de las compensaciones por pérdida de biodiversidad, ligadas a proyectos sujetos a licencia ambiental. Lo anterior, para asegurar la resiliencia de los servicios ecosistémicos al cambio climático, entre otros.

Cumpliendo con lo anterior, el Ministerio desarrolló un esquema de compensaciones con apoyo de expertos y entidades nacionales e internacionales en la materia y expidió, a través de la Resolución 1517 de 2012 el MACPB, que por el momento se enfoca en ecosistemas naturales terrestres continentales y vegetación secundaria y en proyectos sujetos a licenciamiento por parte de la ANLA. Los criterios y metodologías dispuestos en este Manual son de uso obligatorio para un determinado número de sectores, incluyendo el de hidrocarburos, en la elaboración de los estudios de impacto ambiental. La ANLA, por su parte, deberá tomar en consideración el Manual en su proceso de evaluación, aprobación y adopción de las medidas presentadas en el Plan de Compensaciones del proyecto licenciado. Con la aplicación del Manual, se pueden determinar y cuantificar las compensaciones desde la fase de planeación del proyecto a licenciar para establecer cuánto compensar en términos de área, dónde compensar y estrategias sobre cómo compensar. Todo esto se establece siguiendo la jerarquía de la mitigación, es decir, se compensan aquellos impactos a la biodiversidad que no puedan ser primero evitados, mitigados o corregidos por el proyecto.

El gran avance del Manual consiste en haber estandarizado los factores de compensación para cada uno de los biomas y vegetación natural en los ecosistemas terrestres continentales del país, facilitando con ello la identificación del área total a compensar bajo el principio de equivalencia ecosistémica ${ }^{4}$. Los factores individuales de compensación responden a criterios claros de representatividad del ecosistema en el Sistema Nacional de Áreas Protegidas, rareza, remanencia en su estado natural y su tasa de transformación anual (MADS, 2012) ${ }^{5}$. Al final el factor total de compensación para ecosistemas naturales será la sumatoria de los factores individuales donde el valor mínimo del factor total será de cuatro y el máximo de diez. En el caso de la vegetación secundaria menor a 15 años, se aplican los factores de compensación del bioma correspondiente y su rango, el rango de compensación está entre dos y cinco. En ese sentido, un ecosistema como el de los bosques naturales del heliobioma de la Amazonía Orinoquia tendrá un factor total de compensación de siete que deberá multiplicarse con el área impactada de este ecosistema por el desarrollo del proyecto para de esta manera obtener el área a compensar por pérdida de biodiversidad. Así mismo, el factor de compensación total por afectación a vegetación secundaria en este mismo helobioma será de 3.5.

Otro de los puntos innovadores del Manual son los criterios para determinar dónde compensar, estableciendo que las áreas ecológicamente equivalentes deben ubicarse dentro del área de influencia del proyecto o, en su defecto, dentro de la subzona hidrográfica donde se encuentre ubicado. Si esto no fuera posible, deben ubicarse en las subzonas hidrográficas circundantes lo más cerca posible al área impactada. El Manual también establece que las acciones de compensación deben durar hasta por la vida útil del proyecto licenciado.

Una vez determinada el área en extensión y su ubicación, el Manual establece unas estrategias sobre cómo compensar en dicha área. En ese sentido, se podrá optar, dependiendo de las condiciones de los proyectos, por apoyar la creación, ampliación o saneamiento de áreas protegidas del Sistema Nacional de Áreas Protegidas (SINAP) o de las Reservas Naturales de la Sociedad Civil, incluyendo su respectivo plan de manejo. También se podrán llevar a cabo acciones de restauración ecológica en ecosistemas degradados identificados en, por ejemplo, el Plan Nacional de Restauración que establezca el Ministerio de Ambiente.

\section{Problemática y Retos EN LA APLICACIÓN DEL MANUAL}

El MACPB es un gran avance para asegurar el balance del capital natural del país y los beneficios que de allí se derivan para el desarrollo sostenible del país, así como una oportunidad para que el sector de hidrocarburos pueda contribuir efectivamente a partir de una metodología de compensaciones estandarizada y clara.

Sin embargo, a pesar de los avances metodológicos y normativos, existen desafíos importantes tanto para la aplicación del Manual como de los diferentes instrumentos de compensación que existen en el país. Resolverlos es urgente, dadas las proyecciones de crecimiento del sector de hidrocarburos en el país, incluida el área marina, y con

\footnotetext{
4 Por área ecológicamente equivalente o de equivalencia ecosistémica se entiende una de vegetación secundaria o ecosistema natural, que mantiene especies y comunidades similares a los presentes en el ecosistema impactado y que tiene una viabilidad ecológica similar por área, condición y contexto paisajístico.

5 Por ejemplo, el factor de compensación tenderá a incrementarse dentro del rango establecido cuando haya menor representatividad, mayor rareza y mayor tasa de transformación. A su vez, para los biomas con alta o baja remanencia, el factor de compensación será igualmente alto dentro del rango establecido, pues se considera igualmente importante proteger tanto ecosistemas pristinos como relictuales altamente transformados en donde la pérdida adicional de área puede implicar la pérdida de la funcionalidad de los servicios ecosistémicos.
} 
ello la solicitud de licencias ambientales. A junio de 2014, aproximadamente 70 proyectos licenciados por la ANLA deben aplicar esta nueva herramienta, de los cuales 46 son del sector de hidrocarburos (ANLA, 2014). Teniendo en cuenta lo anterior, a continuación se presentan los principales desafíos para lograr capitalizar la oportunidad del nuevo esquema de compensaciones ambientales.

Desafío 1: Generar una oferta de áreas para implementar medidas de compensación ambiental, con empresas y personas especializadas en actividades de conservación y restauración. Las áreas para compensar podrán estar dentro o fuera del SINAP. Cuando el área a compensar está fuera del SINAP, las empresas no encuentran predios aptos y lo suficientemente grandes con propietarios dispuestos a servir de arrendatarios o a vender sus predios (MADS, 2012). Generar una oferta suficiente de áreas disponibles para cumplir con las compensaciones establecidas en la licencia en conservación o restauración, reduciría los tiempos y esfuerzos en la búsqueda de áreas y negociación con los dueños de los predios, disminuyendo por lo tanto los costos transaccionales para las empresas y para las autoridades ambientales. Lo anterior es clave para evitar compensaciones aisladas y desarticuladas. A lo anterior se le debe sumar una oferta suficiente de conocimiento para asegurar que las acciones de conservación o restauración sean de la mejor calidad y maximicen los beneficios ambientales.

Desafío 2: Generar mayor trazabilidad en la implementación de las medidas de compensación, y en la ubicación de las áreas donde se implementan este tipo de actividades. Encuestas realizadas por la ONG Fundepúblico, con información solicitada al Ministerio de Ambiente, la ANLA y la CAR, evidencian que no hay mayor claridad sobre la titularidad y la ubicación de los predios donde se están realizando las compensaciones ambientales (Sarmiento et al, 2014). La información recopilada puso de manifiesto que en la mayoría de los casos las entidades no tienen información sobre el sitio exacto y las características del lugar donde se deben realizar las compensaciones. Del mismo modo, en el estudio antes mencionado realizado por la WCS, se encontró que 90 de los 167 expedientes revisados no incluyen información sobre la ubicación de las áreas en donde se habían implementado las medidas de compensación. con base en información a escalas gruesas y para ecosistemas terrestres continentales. En ese sentido es importante que para futuras actualizaciones del Manual se genere información más precisa sobre el estado de los ecosistemas del país para mejorar los análisis de representatividad, rareza, remanencia y transformación. Así mismo, el Manual requiere de una metodología para determinar los factores de compensación en

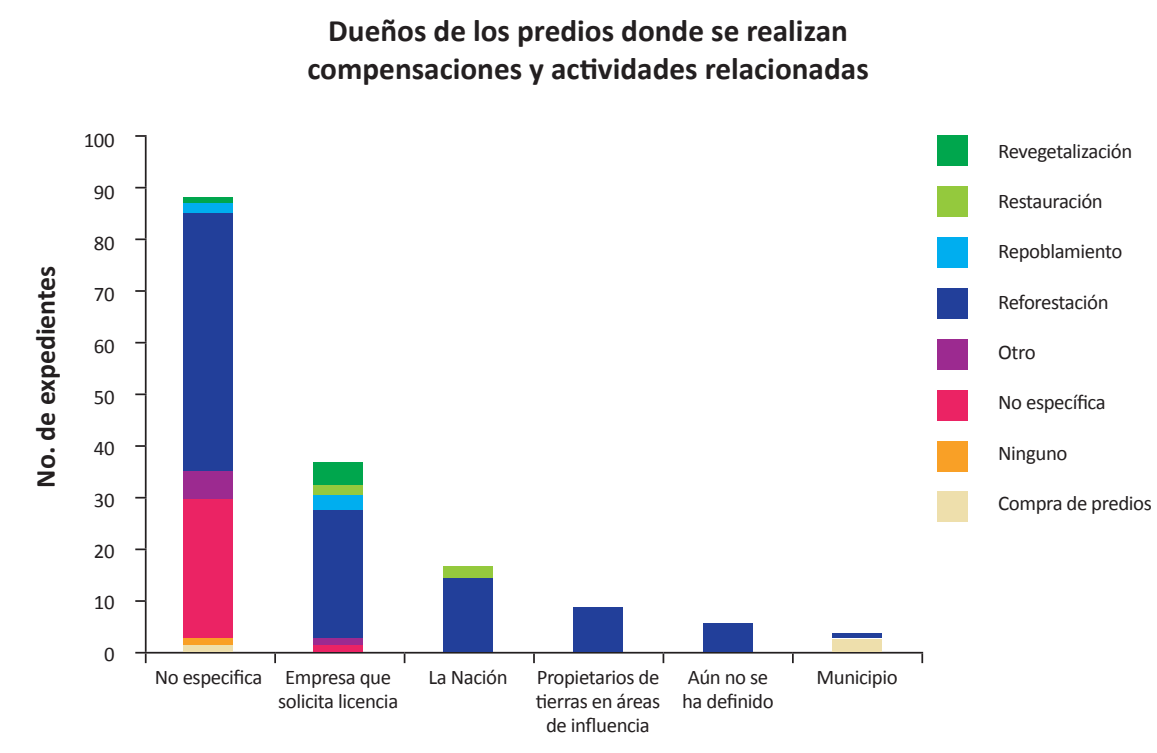

Figura 1. Distribución de los predios objecto de compensaciones Fuente. WCS (2013)

Desafío 3: Lograr la permanencia y la duración de las compensaciones. Si bien el MACB establece el requerimiento de que las compensaciones tengan una duración, equivalente a la vida útil del proyecto, es necesario profundizar los mecanismos jurídicos para asegurar que en las áreas donde se implementa la compensación, las actividades de restauración y conservación perduren en el tiempo. Adicionalmente, es necesario profundizar sobre las implicaciones financieras y los mecanismos de administración de recursos para asegurar la disponibilidad de recursos.

Desafío 4: Generar información que permita una mejor implementación del Manual. Los factores de compensación del Manual fueron elaborados ecosistemas dulciacuícolas y marinos, clave para asegurar la no pérdida neta de biodiversidad y servicios ecosistémicos por parte del sector hidrocarburos.

\section{EXPERIENCIAS INTERNACIONA- LES - LECCIONES APRENDIDAS}

Los anteriores desafíos también se han identificado en otros países. Por tal razón, las lecciones aprendidas a nivel internacional son relevantes para diseñar mecanismos eficientes para la implementación de las compensaciones ambientales. Países como Estados Unidos, Australia, Alemania, Reino Unido y Francia han adoptado mecanismos para asegurar la calidad de las compensaciones, así como para facilitar el desarrollo 
de esquemas costo-eficientes para la implementación de las mismas (Sarmiento et al, 2014). En particular, todos estos países han optado por múltiples mecanismos de implementación que incluyen, entre otros, compensaciones agregadas (donde una sola área suple las necesidades de más de un proyecto) y mecanismos de mercado para facilitar las transacciones entre demanda y oferta de áreas de compensación. Entre estos últimos se encuentran los bancos de hábitat, en donde organizaciones y empresas privadas, de manera anticipada, puedan restaurar, crear o conservar hábitats con el fin de vender los servicios ambientales (en forma de créditos de especies, hábitats o ecosistemas) a uno o varios generadores de impactos ambientales que requieran cumplir con una obligación legal de compensación, siempre y cuando el principio de equivalencia ecosistémica se cumpla (Sarmiento et al, 2014).

Al igual que Colombia, estos países comparten los mismos principios o conceptos adoptados por la nueva normatividad sobre compensaciones por pérdida de biodiversidad: la jerarquía de la mitigación, la no pérdida neta de biodiversidad y la equivalencia ecosistémica. Sin embargo, en estos países se han resuelto algunos aspectos técnicos, legales y financieros que permiten viabilizar los mecanismos antes mencionados (Sarmiento et al, 2014). Dentro de dichos aspectos, se puede resaltar una reglamentación clara sobre los procesos y procedimientos asociados a esquemas agregados de compensaciones. Asimismo, se destacan las figuras legales para garantizar la permanencia de los predios, así como los instrumentos para garantizar la financiación de las medidas de compensación a corto, mediano y largo plazo. A través de estos esquemas se ha facilitado la implementación de las compensaciones y se han logrado los siguientes beneficios:

- La consolidación de empresas o entes especializados en conservación y restauración que ofrecen servicios para el diseño de los planes de compensación y su operación.
- La generación de alternativas económicas para dueños de predios rurales.

- La generación de mecanismos agregados de compensación que maximizan los beneficios ambientales y permiten lograr metas de conservación nacionales y regionales.

- La reducción de los tiempos entre los impactos ambientales ocasionados y la implementación de las medidas de compensación, disminuyendo con ello las pérdidas temporales de biodiversidad y servicios ecosistémicos.

\section{CONCLUSIONES Y PROPUESTAS}

El MACPB logró un avance significativo en la estandarización de los factores de compensación para la determinación del área y de la forma en que estas compensaciones se deben llevar a cabo. Sin embargo, aún hay retos para su implementación y en ese sentido las lecciones aprendidas de otros países pueden contribuir a superar los desafíos ya mencionados. A continuación mencionamos algunas propuestas que consideramos pueden contribuir a superarlos. Esto con el fin de asegurar la sostenibilidad ambiental y financiera de las compensaciones y la permanencia de las mismas. De esta forma se lograría que las compensaciones establecidas en el MACPB puedan efectivamente contribuir al logro de las metas de conservación y restauración y con ello mejorar la oferta de servicios ecosistémicos claves para el desarrollo sostenible del país.

1. Para facilitar la identificación de áreas objeto de restauración como medida de compensación, es importante contar con un Plan Nacional de Restauración que incluya las áreas prioritarias de restauración y sus respectivos protocolos. Así mismo, para las compensaciones en conservación es importante que las autoridades ambientales dispongan de portafolios de áreas prioritarias para la conservación y de necesidades para la consolidación del SINAP.
2. Las autoridades ambientales, junto con la sociedad civil y las empresas, deben generar referentes de buenas prácticas respecto a los contenidos mínimos de un plan de compensaciones. Lo anterior también implica establecer criterios de adicionalidad, calidad y efectividad de las compensaciones para realizar una adecuada evaluación y monitoreo. Lo anterior contribuirá a la unificación de criterios y por lo tanto de reglas claras para el diseño, implementación y evaluación de las medidas de compensación para todos los ejecutores y operadores de las mismas.

3. Se requiere emprender procesos colaborativos entre las empresas privadas, la sociedad civil y las autoridades ambientales, con el fin de implementar proyectos piloto de compensaciones, bajo esquemas agregados, como por ejemplo bancos de hábitat. Lo anterior, para generar esquemas de implementación costo-eficientes para las empresas y para las autoridades ambientales, que incluso permitan articular esquemas de pago por servicios ambientales.

4. Por último, es necesario generar plataformas de acceso público, que faciliten el control y seguimiento a las áreas donde se implementan medidas de compensación ambiental, y también plataformas que faciliten la identificación de posibles áreas o predios donde sea apropiado llevar a cabo dichas compensaciones.

\section{REFERENCIAS}

Agencia Nacional de Hidrocarburos (ANH). (2014). Ronda Colombia 2014. Colombia: The Perfect Environment.

Autoridad Nacional de Licencias Ambientales (ANLA). (Mayo de 2014). s.f. Estrategia para la asignación de compensaciones por pérdida de biodiversidad. Ponencia presentada en Diálogos de Biodiversidad: El Futuro 
de las compensaciones ambientales en Colombia. Bogotá, Colombia.

Business and Biodiversity Offsets Programme (BBOP). (2012). Programa de Negocios y Compensaciones por Pérdida de Biodiversidad (BBOP). Estándar sobre compensaciones por pérdida de biodiversidad. Forest Trends: Washington, D.C.

Decreto 1791 de 1996. Artículo 5. (4 de octubre), por medio del cual se establece el régimen de aprovechamiento forestal. Diario Oficial No. 42,894. Recuperado de http://www. alcaldiabogota.gov.co/sisjur/normas/Norma1.jsp?i=1296

Decreto 2820 de 2010. (5 de agosto), por el cual se reglamenta el Título VIII de la Ley 99 de 1993 sobre licencias ambientales. Diario Oficial. Recuperado de http://www. anla.gov.co/contenido/contenido. aspx?catID=991\&conID=8071
Ministerio de Medio Ambiente y Desarrollo Sostenible (MADS). (2012). Manual para la asignación de compensaciones por pérdida de biodiversidad. Resolución 1517 de 2012, Agosto de 2012. Recuperado de https: //www.siac.gov.co/contenido/contenido.aspx?catID=886\&conID=1389]

Resolución 1517 de 2012, del Ministerio de Medio Ambiente y Desarrollo Sostenible. (2012). Manual para la asignación de compensaciones por pérdida de biodiversidad. Recuperado de https://www. siac.gov.co/contenido/contenido. aspx?catID $=886 \&$ conID $=1389$ ]

Sáenz, S., Walschburger, T., González, J., McKenney, B. y Kiesecker, J. (Diciembre de 2013). Development by Design in Colombia: Making Mitigation Decisions Consistent with Conservation Outcomes, PloS one, 8 (12), e81831. DOI: 10.1371/journal.pone.0081831
Sarmiento, M., López, A., y Mejia, A. (2014). Los bancos de compensación como herramienta de compensación ambiental en Colombia. Documento sin publicar. Bogotá, Colombia.

Unidad de Planeación Minero Energética (UPME). (2012). Escenarios de oferta y demanda de hidrocarburos en Colombia. Ministerio de Minas y Energía. Bogotá. Recuperado de http://www.upme.gov.co/Docs/Publicaciones/2012/Escenarios_Oferta_Demanda_Hidrocarburos.pdf

Wildlife Conservation Society (WCS). (2013). Informe Final Convenio de Asociación 154 de 2013 entre WCS y Ministerio de Ambiente y Desarrollo Sostenible. Diagnóstico sobre la implementación de las compensaciones ambientales en las Corporaciones Autónomas Regionales. Bogotá, Colombia. 